Check for updates

Cite this: J. Mater. Chem. C, 2021, 9, 10547

\section{Solution-processing of semiconducting organic small molecules: what we have learnt from 5,11-bis(triethylsilylethynyl)anthradithiophene}

\author{
Liyang Yu, (D) *a Guiseppe Portale (D) $^{b}$ and Natalie Stingelin (D) *c
}

\begin{abstract}
Organic semiconducting small molecules have attracted increasing interest over the last decades because of their versatile, tunable optoelectronic properties and, e.g., the ease they can be purified compared to polymeric systems. Hence, over the past few decades, a large number of small molecules, such as acenes and thiophenes, have been explored for use in semiconducting devices such as thin-film transistors. However, many of these materials can adopt various molecular arrangements, producing polymorphic structures. As a result, the same material can display vastly different optoelectronic properties. This can, in many cases, lead to a large spread of device performances. Hence, it is critical to establish knowledgeand characterization libraries towards relevant structure/processing/performance interrelations to further advance this interesting class of materials and to open new application platforms. Here, we discuss processing strategies and methodologies that allow the control and assessment of polymorph formation in semiconducting small molecules using 5,11-bis(triethylsilylethynyl)anthradithiophene (TES ADT) as a model material system. We revise how a window into the complex phase behavior of semiconducting small molecules can be obtained, how specific polymorphs can be induced, and how post-deposition treatments can be exploited. Moreover, we illustrate pathways towards patterned structures as needed to fully exploit the touted potential of this interesting class of semiconductors.
\end{abstract}

Received 28th March 2021, Accepted 3rd June 2021

DOI: $10.1039 / \mathrm{d} 1 \mathrm{tc} 01418 \mathrm{~h}$

rsc.li/materials-c

\footnotetext{
${ }^{a}$ School of Chemical Engineering, Sichuan University, Chengdu 610064, P. R. China. E-mail: liyangyu@scu.edu.cn

${ }^{b}$ Zernike Institute for Advanced Materials, University of Groningen, 9747 AG Groningen, The Netherlands

${ }^{c}$ School of Material Science \& Engineering/School of Chemical \& Biomolecular Engineering, Georgia Institute of Technology, Atlanta, GA 30332-0245, USA.

E-mail: natalie.stingelin@gatech.edu
}

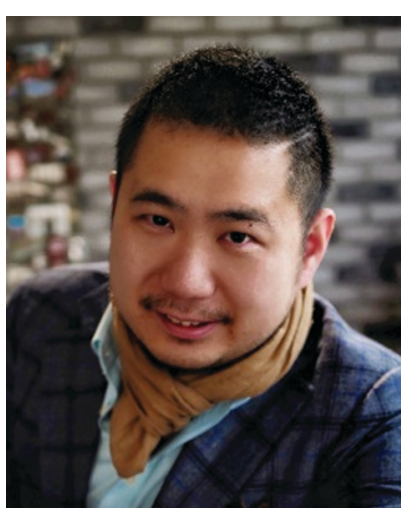

Liyang Yu
Liyang Yu received his PhD degree in 2012 from Imperial College London (ICL) under supervision of Prof. Natalie Stingelin, after which he continued to work as a postdoctoral research assistant at the Centre for Plastic Electronics at ICL for one year. He conducted two further postdoctoral research stays at the King Abdullah University of Science and Technology (KAUST) and Chalmers University of Technology. He joined the College of Chemistry and School of Chemical Engineering at Sichuan University as an Associate Professor in 2019. His research focuses on the processing of organic semiconductors towards applications such as organic solar cells and organic field-effect transistors.

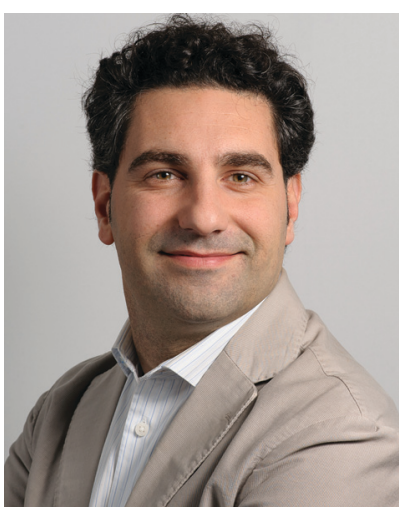

Guiseppe Portale
Giuseppe Portale is associate professor at the Zernike Institute for Advanced Materials, University of Groningen, the Netherlands. He received his $P h D$ degree in Chemistry from the University $\mathrm{La}$ Sapienza, Rome and he carried out postdoc research at the ESRF in Grenoble. From 2009 to 2015 he was beamline responsible at the ESRF and in 2015 he was appointed as professor at the University of Groningen. He is the head of the Polymer Physics group, focusing on the study of structure-property relationship in polymerbased materials and on the influence of processing conditions on the final structure of polymer specimens and devices. 
Observation of semiconducting properties in organic materials was already extensively discussed by, e.g., Eley and Parfitt in the 1950s. ${ }^{1}$ Organic semiconductors have since then raised significant interest both in academia and industry because of their attractive combination of easy-totune optoelectronic properties, low weight, semi-transparency, potential for low-cost and straight-forward processing, to name a few of their desirable attributes. Thereby, both organic small molecules and polymers have been investigated, focusing on device platforms such as field-effect transistors (FET)s, light-emitting diodes (LED)s and photovoltaics (PV)s.

In the semiconducting small molecule area, initial attention was on thermally evaporated systems; however, progressively also solution processable materials, such as substituted acenes, thiophenes and their derivatives (e.g., anthradithiophenes), ${ }^{2-5}$ have been utilized, having led to an increased understanding of this versatile class of materials. Nonetheless, issues are still encountered because of the rich, often highly complex phase behavior of semiconducting small molecules, including the fact that they frequently display various polymorphs as well as metastable phases - a fact that becomes rapidly important in areas such non-fullerene acceptors for organic solar cells, rendering device fabrication often an operator-dominated 'art' rather than being driven by scientific understanding. In this review, we summarize insights collected over the years for 5,11-bis(triethylsilylethynyl)anthradithiophene (TES ADT), first synthesised in the Anthony group, ${ }^{6}$ as an example small molecule semiconductor, with the goal to deliver guidelines and recipes to processing and characterization methodologies that should be broadly applicable across this materials class.

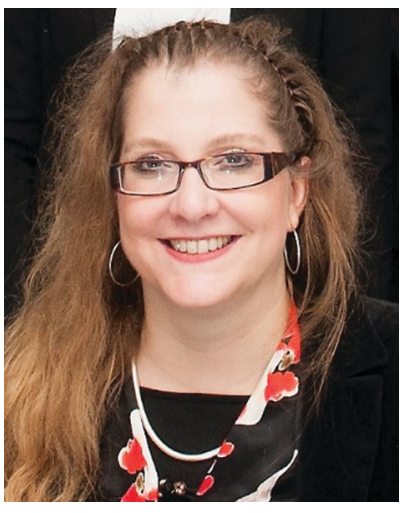

Natalie Stingelin
Natalie Stingelin is a Full Professor at the Georgia Institute of Technology with a joint appointment at the School of Materials Science \& Engineering and the School of Chemical \& Biomolecular Engineering, with prior positions at Imperial College London, UK, at Queen Mary University of London, UK; the Philips Research Laboratories in Eindhoven, The Netherlands; the Cavendish Laboratories, University of Cambridge, UK; and the Swiss Federal Institute of Technology (ETH) Zürich, Switzerland. She is the Co-Director of Georgia Tech's Center of Organic Electronics and Photonics and holds a Chaire Internationale Associee by the Excellence Initiative of the Université de Bordeaux since 2017. She was elected a 2019 Fellow of the Materials Research Society; and is a Fellow of the Royal Society of Chemistry since 2012.

\section{Model system: 5,11-bis (triethylsilylethynyl)anthradithiophene (TES ADT)}

Because of its rich phase behaviour that is well-known and wellstudied, we use in this review TES ADT as case in point to exemplify how phase transformations and the solid-state order of small-molecular semiconductors can be tailored, and how specific polymorphs can be reproducibly induced. Another important aspect that renders TES ADT a useful model material is its excellent charge transport properties. Indeed, TES ADT was the first solution processed organic semiconductor with a charge-carrier mobility, as measured in thin-film field-effect transistors, above $1 \mathrm{~cm}^{2} \mathrm{~V}^{-1} \mathrm{~s}^{-1}$ (see Fig. 1), which was widely considered as the threshold value for practical application of this class of materials and, thus, delivered an important step change towards the technological exploitation of these semiconductors.

Curiously, in the very first report on TES ADT, ${ }^{6}$ this smallmolecular compound was drop-cast from 1-2 wt $\%$ solutions using a relatively slow evaporating solvent (toluene; boiling temperature of $\left.\sim 110{ }^{\circ} \mathrm{C}\right) .{ }^{6}$ During casting, a plastic stick was used to rub the substrate surface to promote crystal nucleation. The reason for employing this rather unusual procedure with apparent drawbacks for larger-area device fabrication and reaching high device reproducibility was that, in most scenarios, TES ADT displays a low tendency to crystallize. For example, when spin coating this material, thin films of
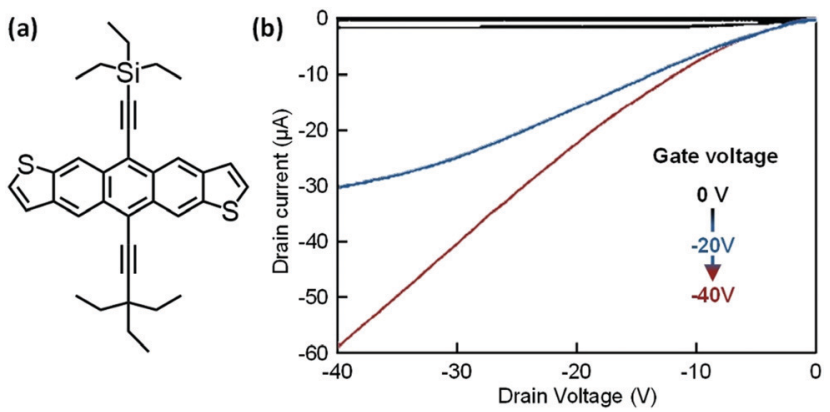

Fig. 1 (a) Chemical structure of the semiconducting small molecule bis(triethylsilylethynyl)anthradithiophene (TES ADT). (b) Output characteristics of the first reported organic field-effect transistor (OFET) fabricated with TES ADT as active material (reproduced with permissions from ref. 6).

Table 1 Unit cell parameters of the four TES ADT crystal structures identified to date in literature (see ref. 6 for the $\alpha$ - and $\beta$-phase; ref. 9 for the $\delta$-phase; and ref. 15 for the $\gamma$-polymorph)

\begin{tabular}{lcclc}
\hline Unit cell parameter & $\alpha$-Phase & $\beta$-Phase & $\gamma$-Phase & $\delta$-Phase \\
\hline$a(\AA)$ & 6.7 & 10.5 & 18.5 & 6.9 \\
$b(\AA)$ & 7.3 & 10.5 & 21.8 & 7.4 \\
$c(\AA)$ & 16.7 & 12.4 & 97.1 & 16.6 \\
$\alpha\left(^{\circ}\right)$ & 98.1 & 90 & 90 & 96 \\
$\beta\left({ }^{\circ}\right)$ & 94.5 & 114.2 & 90 & 96 \\
$\gamma\left({ }^{\circ}\right)$ & 103.9 & 90 & 89.5 & 106
\end{tabular}


predominantly amorphous TES ADT are obtained. ${ }^{7,8}$ However, over the years, a number of different crystalline phases, the $\alpha-, \beta-, \gamma$-, and $\delta$-phase, were reported (see Table 1 ), explaining why charge-carrier mobility values measured in OFETs can spread over six orders of magnitudes. ${ }^{9,10}$ In this review, we will highlight some of the knowledge that has been gained over the years to understand specific aspects of polymorph formation in TES ADT thin films and that now permits TES ADT structures to be deposited over large areas with high reproducibility and to pattern TES ADT in a robust manner.

\section{Phase behavior and phase transformations}

The phase behavior and relevant phase transformations of semiconducting small molecules are not necessarily straightforward to assess as multiple polymorphs may co-exist at a given temperature/pressure (as already alluded to above), and multiple processes (crystallization, solid-solid phase transitions) might occur simultaneously. Differential scanning calorimetry (DSC) can in many situations be a powerful tool to obtain important initial information on such complex polymorphic landscapes and, in particular, on possible phases that can be present at different temperatures and as function of the selected processing conditions.

From the thermograms displayed in Fig. 2a, for instance, the complexity of the phase behavior of TES ADT, when drop-cast at room temperature, immediately becomes clear. ${ }^{10,11}$ In the first heating scan, two endotherms are observed around 135 to $137{ }^{\circ} \mathrm{C}$ and 153 to $155^{\circ} \mathrm{C}$, respectively. The exothermal process occurring between these two endothermal transitions was attributed by Su et al. to the melting of a first crystalline phase (here termed: $\alpha$-phase) that then crystallizes into another polymorph (the TES ADT $\beta$-phase). ${ }^{10}$ This picture is supported by polarized optical microscopy: as-cast films display a spherulitic structure (Fig. 2b, top left panel) while films after heating beyond $137^{\circ} \mathrm{C}$ feature a very different morphology comprised of elongated crystals (Fig. $2 \mathrm{~b}$, top right panel). ${ }^{11}$

Further information can be obtained from such thermal analysis data. Having a closer look at the behavior of the $\beta$-phase, for instance, it was noticed that this TES ADT polymorph can be brought to room temperature and kept stable if, after its formation through melting of the $\alpha$-phase and subsequent crystallization of the material around $140{ }^{\circ} \mathrm{C}$, it is directly cooled without heating it to the isotropic melt, i.e., without heating to temperatures above $\sim 153{ }^{\circ} \mathrm{C}$. In contrast, when heating the TES ADT to the isotropic melt followed by cooling the material to room temperature, no crystallization occurs, even when relatively modest cooling rates of $10{ }^{\circ} \mathrm{C} \mathrm{min}^{-1}$ are used (Fig. 2a). Another crystalline form, the so-called TES ADT $\gamma$-phase may, however, evolve during heating such melt-induced amorphous TES ADT (see Fig. 2a and b, bottom panels), as discussed later in this review.

In materials such as TES ADT that exhibit different polymorphs, seeding can help to induce a specific crystalline form. For instance, the TES ADT $\beta$-phase can be challenging to produce in many scenarios. It does not form when cooling TES ADT from the fully molten state; when heating (temperature annealing) melt-processed amorphous structures; nor when tempering at $140{ }^{\circ} \mathrm{C}$ solvent-vapor annealed films that typically are comprised of the $\alpha$-phase (see Section 4 ). Indeed, the cold crystallization around $140{ }^{\circ} \mathrm{C}$ to the $\beta$-phase is only observed for solution-cast systems (Fig. 2a). Hence, it was hypothesized that the formation of the $\beta$-phase by heating solution-cast $\alpha$-phase thin films is facilitated by $\beta$-phase seeds present in such structures as indicated by the initial X-ray scattering data presented in ref. 6. Such seeds will be erased in the fully molten state, hence, the $\beta$-phase cannot be accessed when melt-processing TES ADT. Usefully, $\beta$-phase seeds can be introduced intentionally, e.g., by pressing $\beta$-phase TES ADT (a)

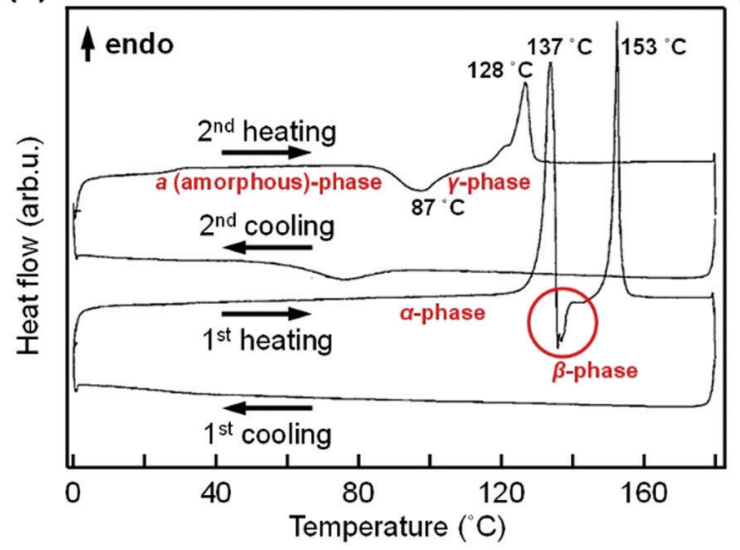

(b)

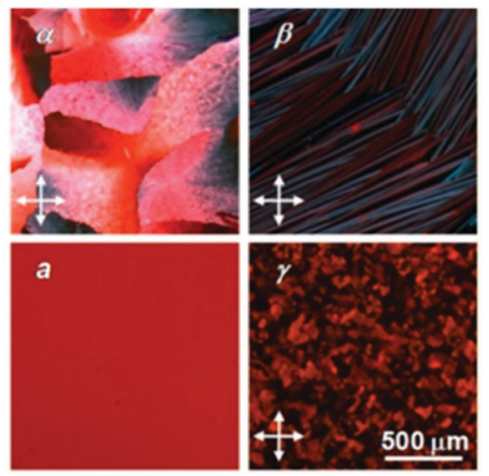

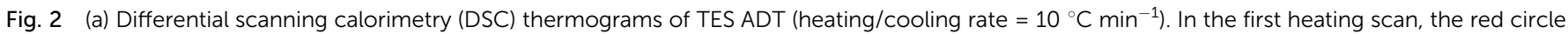

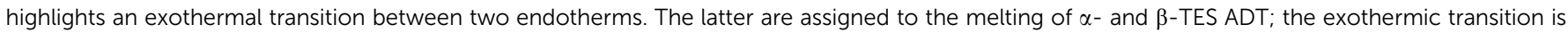

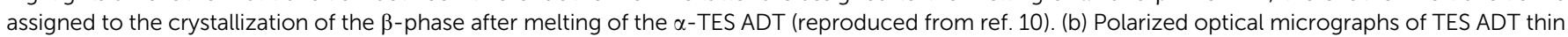
films comprised of the different polymorphs. An amorphous film obtained from the melt is also shown (reproduced with permissions from ref. 11). 
powder at $140{ }^{\circ} \mathrm{C}$ onto melt-induced amorphous structures. This results in films comprised of one dominant polymorph, that is the $\beta$-form, in a relatively straight-forward manner. We note, however, that $\beta$-phase can only be obtained by thermal annealing thin films over a critical thickness which was associated to a two-step nucleation process with influence of the anisotropic surface energy of crystal grains. ${ }^{12}$

In addition to the $\alpha$ - and $\beta$-phases, a third crystalline form can evolve by heating amorphous TES ADT films to temperatures just above $90{ }^{\circ} \mathrm{C}$, as already alluded to in the previous section. At this temperature, an exothermal transition is observed in DSC (Fig. 2a), in agreement with the appearance of a crystalline structure comprised of small, granular domains observed in optical microscopy (Fig. 2b). This TES ADT polymorph, referred to as $\gamma$-phase, melts during heating at $128{ }^{\circ} \mathrm{C}$ (i.e., at lower temperatures than the other two crystalline phases). ${ }^{11}$ It also exhibits significantly lower wide-angle X-ray diffraction intensities than the $\alpha$ - and $\beta$-TES ADT. ${ }^{11}$ In fact, Lee et al. attributed the $\gamma$-phase to a monoclinic crystal structure with diffraction intensities of 1 to 2 orders of magnitude lower than what is observed for the $\alpha$-TES ADT. ${ }^{13}$ Hence, it is not surprising that the $\gamma$-phase was often overlooked and was typically attributed to the amorphous fraction in a given TES ADT structure, ${ }^{14,15}$ even though its charge-carrier mobility (as measured in thin-film transistors) is $>10^{-3} \mathrm{~cm}^{2} \mathrm{~V}^{-1} \mathrm{~s}^{-1}$ compared to $10^{-6} \mathrm{~cm}^{2} \mathrm{~V}^{-1} \mathrm{~s}^{-1}$ measured for fully amorphous thin films. ${ }^{8,11}$

The TES ADT phase behavior is, however, even more complex. Apart from the above described three crystalline forms (i.e., the $\alpha-\beta$ - and $\gamma$-forms), a fourth polymorph of TES ADT was reported by Chen et al., denoted here as $\delta$-phase. ${ }^{9}$ It was produced by solution-casting TES ADT from tetrahydrofuran (THF) at room temperature. The formation of the $\delta$-phase is likely due to the high polarity of the processing solvent, which is of importance as $\delta$-TES ADT displays relatively high charge-carrier mobilities of $10^{-2} \mathrm{~cm}^{2} \mathrm{~V}^{-1} \mathrm{~s}^{-1} \cdot{ }^{9}$

\section{Kinetics of structure formation/ nucleation control to induce favorable polymorphs}

Having a complex material system such as TES ADT that displays a rich phase behavior, with multiple polymorphs having been identified, it is important to gain insights into the kinetics of how these crystalline forms evolve and how this process can be manipulated. We first focus here on the $\alpha$-phase, because it features the best charge-transport properties compared to all other polymorphs, roughly 2 to 3 orders of magnitude higher than the ones observed for the $\beta$-phase ${ }^{11}$ or $\delta$-TES ADT. ${ }^{9}$ Tellingly, when keeping amorphous TES ADT films at room temperature, the $\alpha$-phase starts to evolve after seven days and longer, ${ }^{8}$ in line with the fact that the glass transition temperature of TES ADT was reported to be around $27{ }^{\circ} \mathrm{C},{ }^{7}$ which can be assumed to enable some mass transport at room temperature and, in turn, slow crystallization into the $\alpha$-crystalline form.

Beneficially, this process can be accelerated via nucleation. Here we discuss two pathways: (i) promoting nucleation through judicious selection of processing conditions; and (ii) use of nucleation agents. More specifically, since homogenous nucleation is controlled by thermodynamic driving forces and molecular diffusion processes, ${ }^{11}$ during solution deposition of organic small molecules such as TES ADT, nucleation can be induced by keeping the system at a supersaturated state for an extended time. This often requires a relatively slow process in case of highly soluble materials such as TES ADT because supersaturation must be reached during solvent evaporation, i.e., prior to complete drying. To give an example, in case of TES ADT, $4 \mathrm{wt} \%$ solutions in chloroform can be used and cast at relatively low temperatures $\left(5^{\circ} \mathrm{C}\right)$ to produce homogenous films comprised of the $\alpha$-phase in highly reproducible fashion in a convenient, one-step drop casting approach (Fig. 3a). ${ }^{7}$ Thereby, the low casting temperature, assists in reducing the solvent (a)

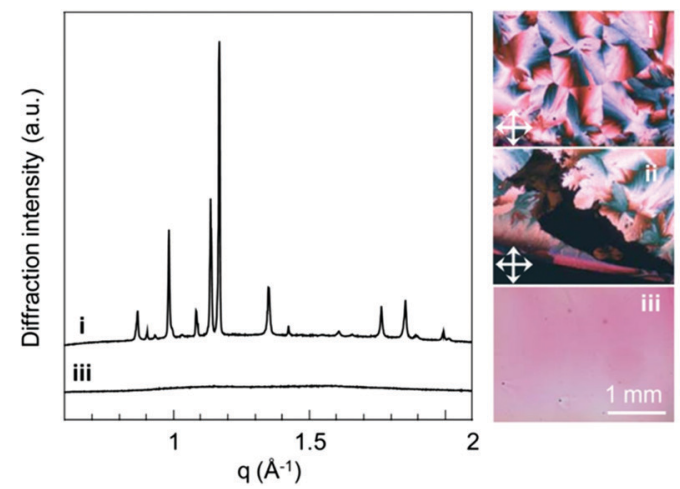

(b)

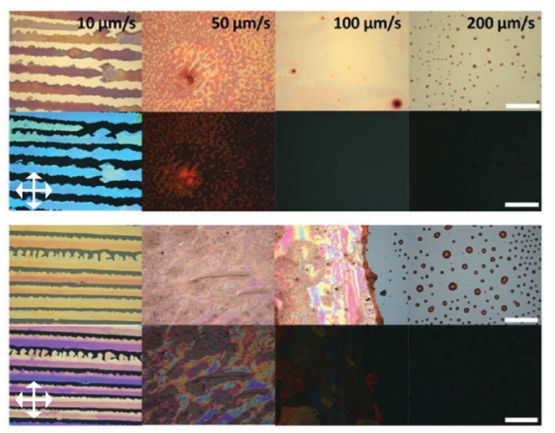

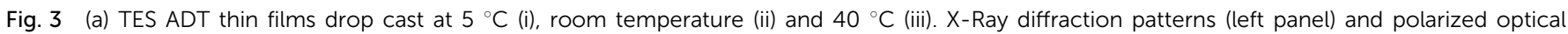

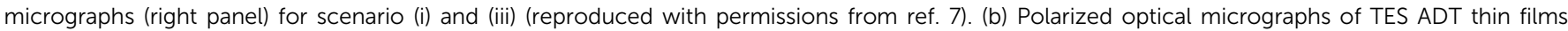

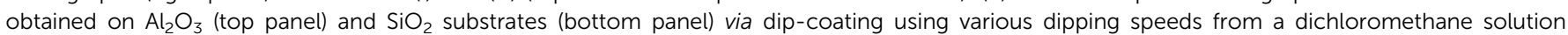
(reproduced with permissions from ref. 17). 
evaporation rate and, thus, prolonging time for nucleation and crystal growth to occur, even when lowering overall mass transport.

Supersaturation of a solution can of course be controlled via selection of concentration and the materials solubility in a given solvent. This can be exemplified by the fact that homogeneous $\alpha$-TES ADT films were also obtained when casting from toluene solutions and using an even higher TES ADT concentration (8 wt\%). In toluene, TES ADT has a moderate solubility, ${ }^{16}$ hence an increase in TES ADT concentration will result in the solution to reach superstation relatively fast. This is important to ensure that the desired nucleation and growth processes can proceed while the system is in the liquid state, i.e., in a state where diffusion can occur. Other solution processing methods, such as dip coating, can potentially be used to further retard the drying kinetics. Ribbon-like crystals were, for instance, obtained using this methodology by moving the substrate at a speed of as low as $10 \mu \mathrm{m} \mathrm{s}^{-1}$ from a solution of $0.7 \mathrm{wt} \%$ TES ADT in dichloromethane (Fig. $3 \mathrm{~b}){ }^{17}$

In many situations it may be useful to exploit heterogeneous, rather than homogeneous, nucleation to accelerate crystallization. This can be achieved via use of nucleation agents. Thereby, various species can act as nucleating agents - such as materials with a lower solubility in a given solvent than the material of interest. For example, adding a small amount of 2,8-difluoro-5,11bis(triethylsilylethynyl)anthradithiophene (diF-TES ADT), a fluorinated derivative of TES ADT with poorer solubility than the latter in most solvents, has been shown to assist nucleation of $\alpha$-TES ADT thin films. This is evident from the observed progressive decrease of spherulitic domain size with increasing amount of diF-TES ADT content in TES ADT thin films. ${ }^{18}$ However, it has to be noted that these nucleation sites did not induce crystalline $\alpha$-TES ADT directly from solution. Rather, solvent vapor annealing (SVA) was required, likely because molecular mobility is needed to enable diffusion to assist crystal growth (see Section 3 for post-deposition treatments).

Commercial nucleating agents can also be applied to heterogeneously nucleate organic semiconductors. This can, e.g., be illustrated by the use of nucleating agents that were designed for the controlled melt solidification of isotactic polypropylene (i-PP), e.g., tris-tert-butyl-1,3,5-benzenetrisamide (BTA; Irgaclear XT 386), ${ }^{19}$ which has been demonstrated to be useful to manipulate the solidification of 6,13-bis(triisopropylsilylethynyl) pentacene (TIPS pentacene) - a material that chemically has many similarities to TES ADT (Fig. $4 \mathrm{~b}$ and c). This led to homogenous film formation and, beneficially, minimized dewetting. Nucleating agents, thus, enabled the fabrication of thin-film transistors with uniform electrical characteristics at high yield and allowed straight-forward ink jet printing of this important semiconducting small molecules. ${ }^{20}$

\section{Post-deposition treatments}

The strong tendency of TES ADT to form amorphous films when deposited from solution can be advantageous. In fact, it (a)

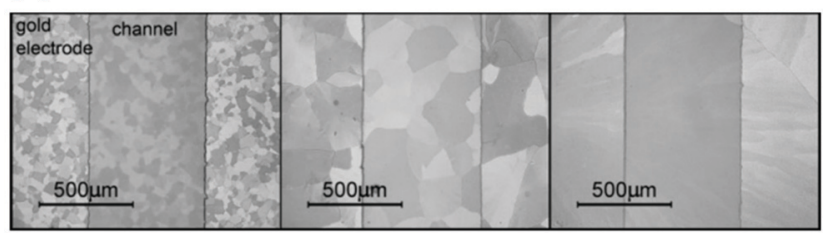

(b)

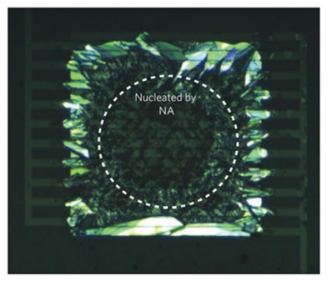

(c)

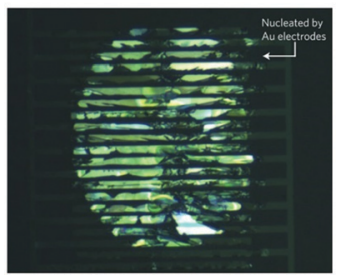

Fig. 4 (a) Optical micrographs of the channel region of OFET devices fabricated with TES ADT comprising different amounts of diF-TES ADT as nucleating agent. Shown are blends with $1.17 \mathrm{~mol} \%$ (left), $0.95 \mathrm{~mol} \%$ (middle) and $0.63 \mathrm{~mol} \%$ (right) diF-TES ADT (reproduced with permissions from ref. 18). ( $b$ and c) Polarized optical micrographs of OFET devices produced by ink-jet printing TIPS-pentacene with (b) and without (c) commercially available nucleation agents used in commodity plastic manufacturing (reproduced with permissions from ref. 20).

has been shown on the example of rubrene that inducing first vitrified structures and then crystallizing them at a later stage can assist in producing specific crystal forms in a highly robust manner. ${ }^{21}$ The reason is that production of initially amorphous films allows in many cases precise control of, e.g., crystallization temperature and film thickness. This approach, however, requires that post-deposition treatments be accurately manipulated to induce the desired polymorph and to ensure that, e.g., targeted domain sizes be obtained. In case of TES ADT, the initially amorphous films can be crystallized into the desired $\alpha$-phase after three days in vacuum (room temperature); films were fully crystallized after seven days (Fig. 5a). ${ }^{8}$

Clearly, such time-consuming methods are not ideal. To accelerate the transformation to the desired crystalline form, therefore, solvent vapor annealing may be explored as postdeposition treatment, as already discussed above. The advantage of using a SVA process is that it can promote crystallization at room temperature as opposed to thermal annealing. The reason is that exposure to solvents will lead to (partial) swelling of the organic thin films, assisting mass transport and, hence, diffusion. As a consequence, the time needed for crystal growth is drastically reduced (Fig. 5b). This can be illustrated by work by Dickey et al. who placed amorphous thin films of TES ADT into various solventvapor environments, identifying 1,2-dichloroethane as the most suitable solvent and observing the films to start crystallizing within minutes rather than days. ${ }^{14}$ The gained speed in process did, however, come at a cost: the obtained structures displayed typically charge-carrier mobilities that were lower than those obtained for films where the $\alpha$-phase was directly induced from solution, 6,7 although mobilities of $\sim 0.4 \mathrm{~cm}^{2} \mathrm{~V}^{-1} \mathrm{~s}^{-1}$ could be induced via SVA using chloroform as solvent. ${ }^{15}$

Other, higher-mobility TES ADT structures were obtained combining blending with an insulating polymer and SVA using 
(a)

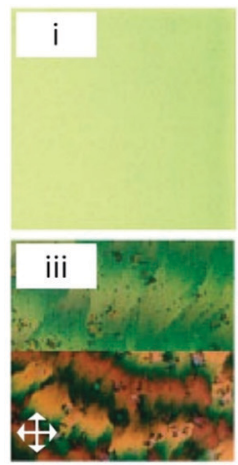

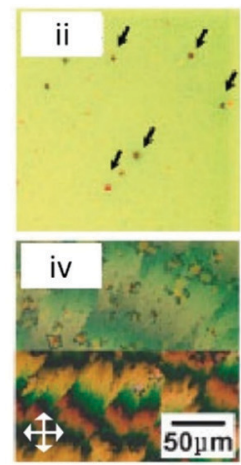

(b)
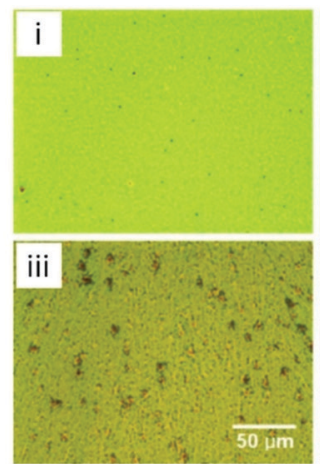

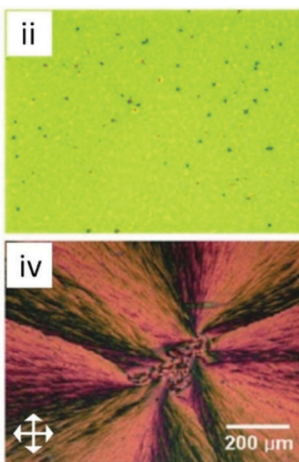

Fig. 5 (a) Optical micrographs of TES ADT thin films: as cast (i) and kept at room temperature and vacuum for 3 days (ii), 5 days (iii) and 7 days (iv), respectively (reproduced with permissions from ref. 8). (b) Optical micrographs of TES ADT thin films kept in a solvent vapor rich environment for (i) 3 min, (ii) $10 \mathrm{~min}$, and (iii) $30 \mathrm{~min}$. For comparison, also a polarized optical micrograph of a thin film annealed for 30 min is shown (iv) (reproduced with permissions from ref. 15).

1,2-dichloroethane vapour. ${ }^{22}$ In these experiments, a thermalannealing step around $90{ }^{\circ} \mathrm{C}$ was used prior to the SVA steps. Such temperatures are sufficiently high to transfer the amorphous structure into the $\gamma$-phase, ${ }^{11}$ which may explain the increase in electronic performance of these structures compared to samples not comprising the macromolecular additive. Clear is that introduction of the polymer assists with dewetting issues often encountered when processing semiconducting small molecules, thus, providing a simple two-step method for the fabrication of homogenous, crystalline and high charge-carrier mobility TES ADT films at high yield. ${ }^{23}$

\section{Towards structure/property relationships}

Having a material where specific polymorphs can be induced throughout an entire film assists to obtain some insights on how structure dictates certain properties. When, for instance, comparing $\alpha$ - and $\beta$-TES ADT, which both have a similar density and feature similar diffraction intensities (indicating a similar degree of molecular order), ${ }^{11}$ the $\alpha$-phase displays chargecarrier mobilities that are two to three orders of magnitude higher than those measured for the $\beta$-phase. ${ }^{11}$

Such dramatic differences in properties are a direct consequence of changes in molecular packing. Especially for organic small molecules, such as TES ADT, that are comprised of linear fused rings, a small variation in molecular packing can lead to large deviations in charge transport. ${ }^{24-26}$ The schematic unit cells of the $\alpha$ - and $\beta$-phase are provided in Fig. 6 as an example. The $\alpha$-phase features a clear 2-D $\pi$-stacking arrangement similar to other high charge-transport small molecules. ${ }^{27-29}$ In contrast, the $\beta$-phase displays a packing style with very limited overlap between the $\pi$-orbitals of neighboring TES ADT molecules, explaining why this polymorph features even smaller charge-carrier mobilities than the $\gamma$-phase, which clearly is of much lower molecular order as one can determine from the significantly lower enthalpies of fusion that are observed for $\gamma$-TES ADT in DSC, and the low intensities that are measured for this phase in X-ray diffraction. ${ }^{11}$
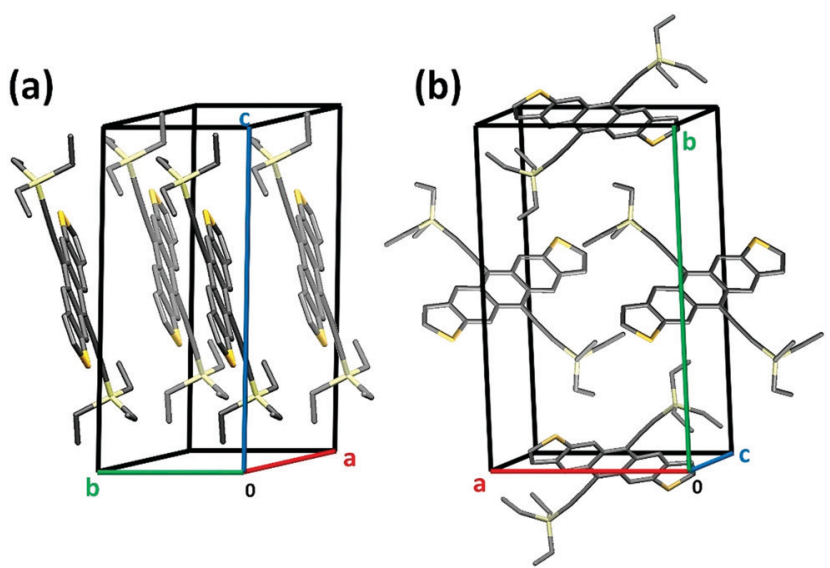

Fig. 6 Crystalline packing in $\alpha$ - (a) and $\beta$-TES ADT (b).

Other structure/property relations can be established, e.g., with respect to how grain boundaries influence charge transport. Most noteworthy here is work by Lee $e t$ al. who produced TES ADT structures comprised of large spherulites and who compared the charge transport properties in the radial and azimuthal axis within a single spherulite (Fig. 7a). ${ }^{30}$ Interestingly, it was found that the charge transport property is almost independent of the direction of charge transport within a TES ADT spherulite with saturated mobilities recorded being between 0.3 to $0.4 \mathrm{~cm}^{2} \mathrm{~V}^{-1} \mathrm{~s}^{-1}$ regardless of the angle between the FET channel and the local spherulite growth axis. This is surprising as similar molecules often exhibit at least a two-fold difference in transport along different molecular packing directions. ${ }^{31-33}$ Clearly, this is a highly desirable attribute, especially if such TES ADT architectures were to be integrated into electronic circuits. The size of the spherulites or grains plays, however, an important role. Numerous studies have, for instance, demonstrated that when grain/spherulite size are decreased (e.g., through introduction of heterogeneous nucleating sites) an increase in the number of grain boundaries is observed and, in turn, reduced charge transport properties are measured. ${ }^{18,34,35}$ For example, by controlling the crystal size by tuning the solvent vapor annealing 
(a)
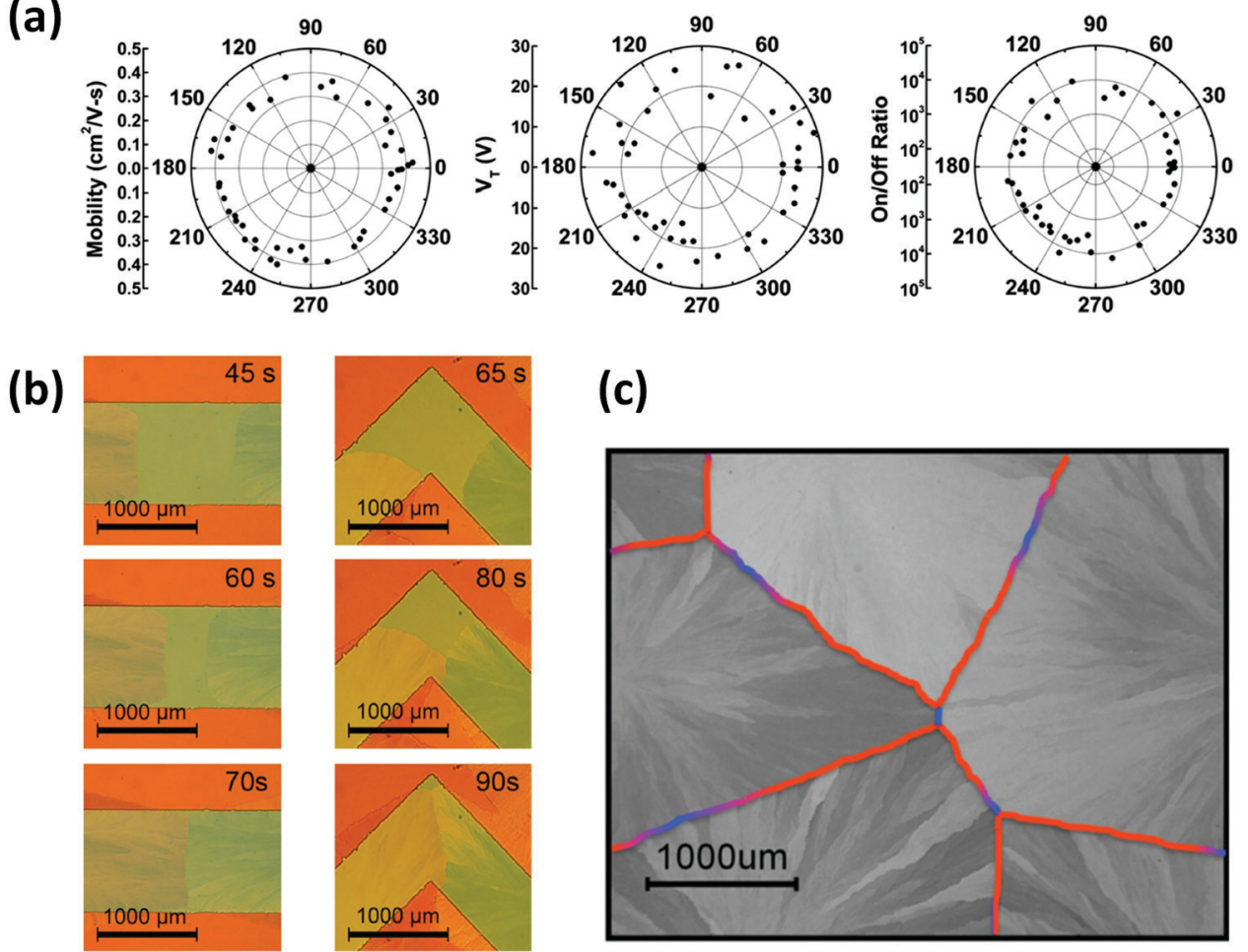

(c)

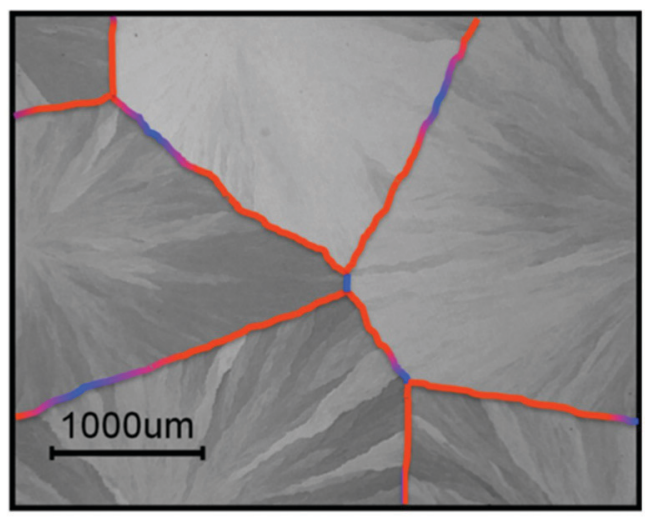

Fig. 7 (a) Pole figures showing the low directional dependence of transistor mobility, threshold voltage and on-off ratio measured within one single TES ADT spherulite (reproduced with permissions from ref. 30). (b) Optical micrographs of TES ADT thin films deposited on surface-energy patterned substrates illustrating formation of high-angle and low-angle inter-spherulite boundaries. (c) Optical micrograph of a TES ADT thin film where high-angle (blue) and low-angle inter-spherulite boundaries (red) are readily identified (reproduced with permissions from ref. 36).

conditions, charge-carrier mobilities over $1 \mathrm{~cm}^{2} \mathrm{~V}^{-1} \mathrm{~s}^{-1}$ were achieved in crystals that were significantly larger than the device channel length, while for crystals that were smaller than the channel, leading to grain boundaries in the channel, mobilities of $0.3 \mathrm{~cm}^{2} \mathrm{~V}^{-1} \mathrm{~s}^{-1}$ were recorded. ${ }^{34}$

This issue can be circumvented by introducing "soft grain boundaries", achieved by directing crystal growth in a patterned channel (Fig. $7 \mathrm{~b}$ and c). ${ }^{36}$ Such structures featured very different charge transport characteristics compared to material crystallized directly from solution or via vapor-phase deposition, without crystal growth restrictions. ${ }^{37,38}$ Accordingly, the authors could distinguish between low- and high-angle interspherulite boundaries. In the low-angle situation, the nearly fully aligned crystals in neighboring spherulites led to essentially unaffected charge transport across the grain boundary $(R=$ $1.8 \pm 0.1 \Omega$, compared to $R=1.6 \pm 0.1 \Omega$ within one crystalline domain), in contrast to high-angle inter-spherulite boundaries with large geometrical mismatch that resulted in a large barrier for charge transport across such boundaries $(R=2.7 \pm 0.3 \Omega) .{ }^{36}$

\section{Crystal patterning}

Generating patterned structure is vital when, e.g., integrating transistors into circuits as it can reduce, if not fully eliminate, parasitic leakage currents; it may also lower OFF-currents. Arranging crystals into specific patterns moreover can avoid grain-boundary formation in the device channel or selectively induce high-angle inter-spherulite boundaries, ${ }^{35}$ thus, preventing a decrease in charge transport in the device.

While conventional ink-jet printing or vacuum deposition through a shadow mask are often-investigated approaches towards patterned structures, spatially controlled crystallization can provide an interesting alternative. For this, spatial control of substrate surface energy may be exploited because the energy landscape at the growth front is altered. ${ }^{39}$ Lee et al. observed, for instance, that the crystal growth rate is affected by variations in the substrate surface energy ${ }^{13}$ independent whether the material was deposited from solution ${ }^{40,41}$ or from the vapor phase. ${ }^{42,43}$ In case of TES ADT, surface energy patterns enabled directed, fast crystal growth through a $\mathrm{SiO}_{2}$ channel placed between two $\mathrm{Au}$ pads treated with selfassembled pentafluorobenzenethiol (PFBT) monolayers, with crystals growing within curved channels and along corners (Fig. 7b) ${ }^{13}$ This work also enabled elucidation of the effect on charge transport of low- and high-angle inter-shperulite boundaries (discussed in the previous section), ${ }^{36}$ as well as the influence of capillary effects on the crystal growth along the channels. $^{44}$

Attempts of creating patterned structures in more direct ways have also been explored by degrading parts of a crystallized film via exposure to UV light or removing parts of the film by absorption to a solvent-soaked poly(dimethylsiloxane) (PDMS) stamp. ${ }^{45}$ More elegantly, solvent-soaked PDMS stamps may be 
(a)
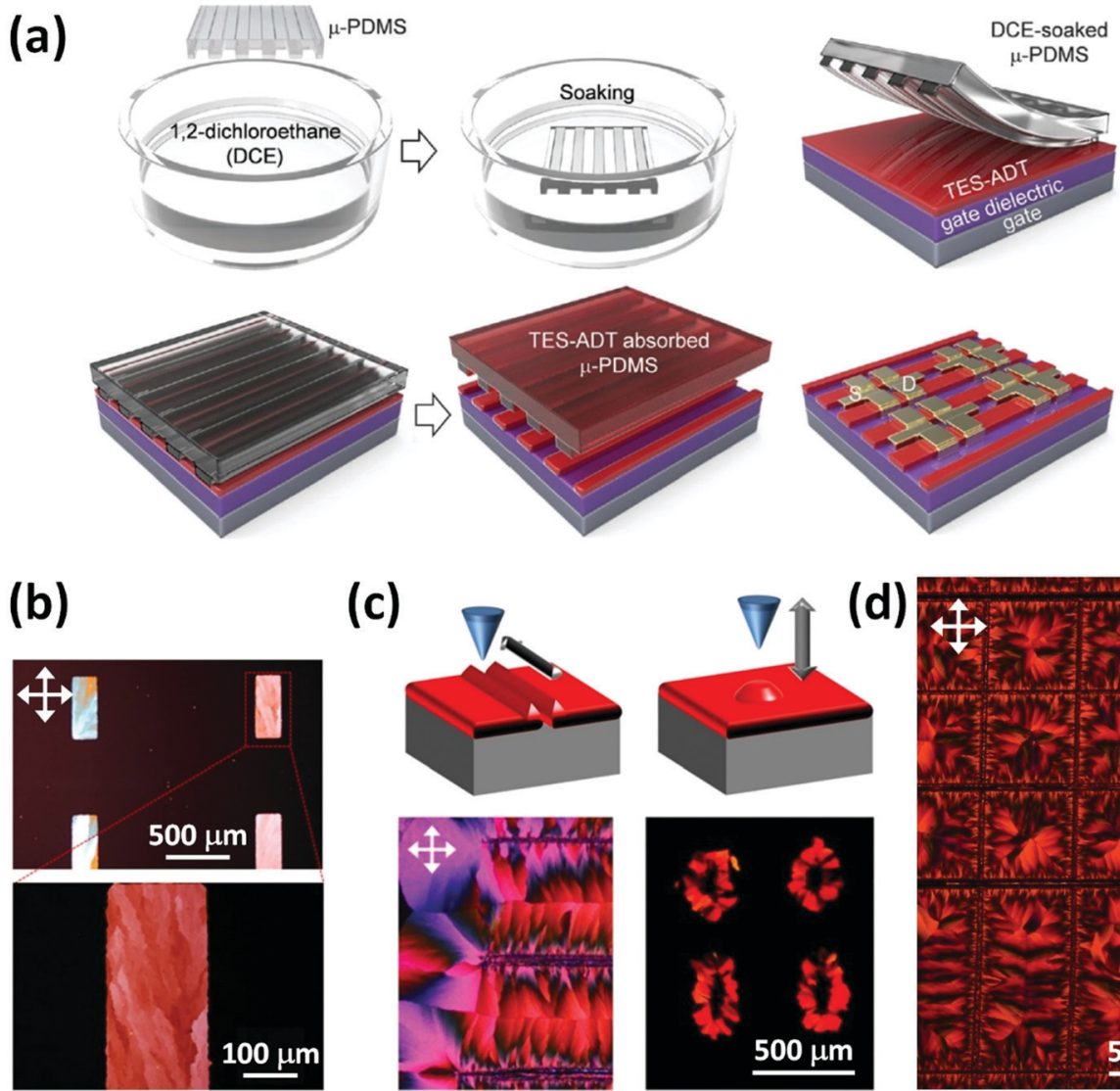

(c)
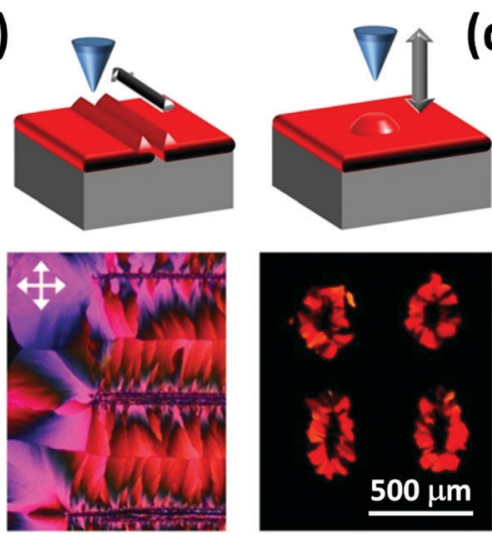

(d)

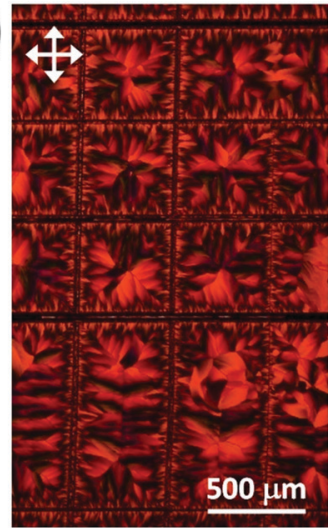

Fig. 8 (a) Schematic outlining the patterned crystallization process via use of a wet PDMS stamp (reproduced from ref. 46). (b) Optical micrographs of a thin film comprising rectangular TES ADT patterns fabricated using a wet PDMS stamp (reproduced with permissions from ref. 47). (c) Illustration of how nucleation can be initiated by mechanical contact (top panels); and optical micrographs of such patterned films (bottom panels). (d) Optical micrograph of "inversed" rectangular spherulites of various sizes produced by controlled nucleation induced in a grid pattern (reproduced with permissions from ref. 23).

used both to remove parts of a given, amorphous TES ADT film structure while simultaneously delivering solvent to specific areas in order to solvent-vapor anneal them (Fig. 8a). Accordingly, high-angle inter-spherulite boundaries are induced where the stamp was not in contact with the TES ADT film, especially when the width of grooves was reduced to around $50 \mu \mathrm{m}$. Note that no grain boundaries were detected when groove widths of $2.5 \mu \mathrm{m}$ were used, ${ }^{46}$ indicating some meso-epitaxial growth mechanisms. This view is supported by the work by Nguyen et al. who reported a similar method based on the use of wet 2-D patterned PDMS stamps that led to the creation of isolated rectangular patterns of crystallized material (Fig. 8b) with apparent advantage for circuits design. ${ }^{47}$

Finally, we like to highlight that the nucleation can also be initiated at pre-designed locations. This can be achieved, for instance, by varying the local thickness of amorphous thin films through mechanical pressing (Fig. 8c). ${ }^{23}$ Since crystallization can be initiated via a mechanical stimulus, especially in thicker areas (simply because in those regions it is more likely that nuclei are present), crystal orientation can be dictated to match, for example, circuit design requirements (Fig. 8d). Such directed crystal orientation may also be exploited to induce good charge-transport regions; indeed, in such TES ADT structures, average mobilities of $1 \mathrm{~cm}^{2} \mathrm{~V}^{-1} \mathrm{~s}^{-1}$ were recorded, likely due to the reduction of the amount of "hard" grain boundaries, with almost negligible device to device variation. ${ }^{23}$

\section{Conclusions and outlook}

TES ADT, which is a promising candidate for future organic electronics applications, was used here to provide a window into the sophisticated and complex phase behavior that semiconducting small molecules can display. In the very first report ${ }^{6}$ on TES ADT, field-effect transistors were produced with mobilities $>1 \mathrm{~cm}^{2} \mathrm{~V}^{-1} \mathrm{~s}^{-1}$, leading to extensive research in the following decades centered around this promising semiconducting small molecule to establish reproducible processing routes, to reveal critical structure/property relationships, to develop patterning methods for creating sophisticated structures, and to advance new materials based on similar design motifs as TES ADT.

We revisited here critical phenomena dictating crystallization of such materials and, specifically, the formation of different polymorphs. We used insights that were obtained on 
the different TES ADT crystalline forms to establish correlations between structure and properties (with focus on charge transport) with the view to guide future processing design towards producing, in robust and reliable ways, desired architectures. As importantly, processing methods explored for creating highperformance thin-film structures of TES ADT in a highly reproducible fashion were revised including solution processing methods, post treatments and crystal patterning. We thus used TES ADT as model system to provide inspiration for how solution processing techniques can be exploited for obtaining desired thin film structures from semiconducting small molecules.

We like to end with highlighting that the future of semiconducting small molecules is very promising. The recent, successful synthesis of, e.g., pure isomers of TES ADT, ${ }^{48}$ namely anti- and syn-TES ADT, inspired new research on the phase behavior $^{49}$ and structure/property relationship ${ }^{50}$ of semiconducting small molecules. Indeed, the discovery of isomeric guest-host systems of TES ADT using blends of the two pure isomers provided further, detailed insight on the polymorph development of TES ADT. ${ }^{51}$ Apart from high performance transistors, moreover, new applications of TES ADT are being explored, including gas sensors ${ }^{52,53}$ and the use of this semiconducting small molecule in photon-upconversion. ${ }^{54}$ These new research frontiers open new opportunities for TES ADT and other semiconducting small molecules and requires further insights into their solidification and controlled fabrication towards specific structures produced for targeted applications.

Since polymorphism have been observed in various solution-processable semiconducting small molecules ${ }^{55,56}$ and polymers, ${ }^{57,58}$ it is unwise to ignore the potential for manipulating the performance of organic electronic devices via control of their solid-state structure/crystal formation. Amongst the various insights gained with TES ADT, key lessons applicable to other systems include the importance to gain detailed understanding of the phase behavior of a given material, including information on possible polymorphs that may form, so that relevant structure/property relationships can be established with respect to device performance. This also requires to advance and develop suitable processing routes and conditions that permit inducing the desirable polymorph(s) and that enable thin-film patterning by taking advantages of the performance disparity of various polymorphs, towards better, more reliable devices and entirely new applications.

\section{Conflicts of interest}

There are no conflicts to declare.

\section{Acknowledgements}

L. Y. thanks the National Natural Science Foundation of China (NSFC \#21905185) for financial support, N. S. is grateful for funding from the U.S. National Science Foundation (NSF) within NSF's DMR program (project \#1905901). The ESRF is acknowledged for providing beamtime for structural analysis.

\section{References}

1 D. D. Eley and G. D. Parfitt, Trans. Faraday Soc., 1955, 51, 1529.

2 H. Klauk, Chem. Soc. Rev., 2010, 39, 2643.

3 H. Sirringhaus, Adv. Mater., 2014, 26, 1319-1335.

4 J. E. Anthony, Angew. Chem., Int. Ed., 2008, 47, 452-483.

5 C. Reese, M. Roberts, M. Ling and Z. Bao, Mater. Today, 2004, 7, 20-27.

6 M. M. Payne, S. R. Parkin, J. E. Anthony, C. Kuo and T. N. Jackson, J. Am. Chem. Soc., 2005, 127, 4986-4987.

7 L. Yu, X. Li, E. Pavlica, M. A. Loth, J. E. Anthony, G. Bratina, C. Kjellander, G. Gelinck and N. Stingelin, Appl. Phys. Lett., 2011, 99, 5-8.

8 W. H. Lee, J. A. Lim, D. H. Kim, J. H. Cho, Y. Jang, Y. H. Kim, J. I. Han and K. Cho, Adv. Funct. Mater., 2008, 18, 560-565.

9 J. Chen, M. Shao, K. Xiao, A. J. Rondinone, Y.-L. Loo, P. R. C. Kent, B. G. Sumpter, D. Li, J. K. Keum, P. J. Diemer, J. E. Anthony, O. D. Jurchescu and J. Huang, Nanoscale, 2014, 6, 449-456.

10 Y. Su, L. Zheng, J. Liu and Y. Han, RSC Adv., 2013, 3, 5529. 11 L. Yu, X. Li, E. Pavlica, F. Koch, G. Portale, I. Da Silva, M. A. Loth, J. E. Anthony, P. Smith, G. Bratina, B. K. C. Kjellander, C. W. M. Bastiaansen, D. J. Broer, G. H. Gelinck and N. Stingelin, Chem. Mater., 2013, 25, 1823-1828.

12 J. Rozbořil, K. Broch, R. Resel, O. Caha, F. Münz, P. Mikulík, J. E. Anthony, H. Sirringhaus and J. Novák, Cryst. Growth Des., 2019, 19, 3777-3784.

13 S. S. Lee, S. B. Tang, D. M. Smilgies, A. R. Woll, M. A. Loth, J. M. Mativetsky, J. E. Anthony and Y. L. Loo, Adv. Mater., 2012, 24, 2692-2698.

14 K. C. Dickey, J. E. Anthony and Y.-L. Loo, Adv. Mater., 2006, 18, 1721-1726.

15 W. H. Lee, D. H. Kim, J. H. Cho, Y. Jang, J. A. Lim, D. Kwak and K. Cho, Appl. Phys. Lett., 2007, 91, 30-32.

16 H. T. Yi, M. M. Payne, J. E. Anthony and V. Podzorov, Nat. Commun., 2012, 3, 1259.

17 S. Nam, J. Jang, J. E. Anthony, J. J. Park, C. E. Park and K. Kim, ACS Appl. Mater. Interfaces, 2013, 5, 2146-2154.

18 S. S. Lee, C. S. Kim, E. D. Gomez, B. Purushothaman, M. F. Toney, C. Wang, A. Hexemer, J. E. Anthony and Y.-L. L. Loo, Adv. Mater., 2009, 21, 3605-3609.

19 F. Abraham, S. Ganzleben, D. Hanft, P. Smith and H. Schmidt, Macromol. Chem. Phys., 2010, 211, 171-181.

20 N. D. Treat, J. A. Nekuda Malik, O. Reid, L. Yu, C. G. Shuttle, G. Rumbles, C. J. Hawker, M. L. Chabinyc, P. Smith and N. Stingelin, Nat. Mater., 2013, 12, 628-633.

21 N. Stingelin-Stutzmann, E. Smits, H. Wondergem, C. Tanase, P. Blom, P. Smith and D. de Leeuw, Nat. Mater., 2005, 4, 601-606.

22 W. H. Lee, J. A. Lim, D. Kwak, J. H. Cho, H. S. Lee, H. H. Choi and K. Cho, Adv. Mater., 2009, 21, 4243-4248.

23 L. Yu, M. R. Niazi, G. O. Ngongang Ndjawa, R. Li, A. R. Kirmani, R. Munir, A. H. Balawi, F. Laquai and A. Amassian, Sci. Adv., 2017, 3, e1602462. 
24 G. Giri, R. Li, D.-M. Smilgies, E. Q. Q. Li, Y. Diao, K. M. M. Lenn, M. Chiu, D. W. W. Lin, R. Allen, J. Reinspach, S. C. B. C. B. Mannsfeld, S. T. T. Thoroddsen, P. Clancy, Z. Bao and A. Amassian, Nat. Commun., 2014, 5, 3573.

25 J. Smith, W. Zhang, R. Sougrat, K. Zhao, R. Li, D. Cha, A. Amassian, M. Heeney, I. McCulloch and T. D. Anthopoulos, Adv. Mater., 2012, 24, 2441-2446.

26 A. Troisi and G. Orlandi, J. Phys. Chem. B, 2005, 109, 1849-1856.

27 O. Jurchescu, D. Mourey, S. Subramanian, S. Parkin, B. Vogel, J. Anthony, T. Jackson and D. Gundlach, Phys. Rev. B: Condens. Matter Mater. Phys., 2009, 80, 085201-085207.

28 T. Izawa, E. Miyazaki and K. Takimiya, Adv. Mater., 2008, 20, 3388-3392.

29 C. D. C. D. Sheraw, T. N. T. N. Jackson, D. L. D. L. D. L. Eaton and J. E. J. E. J. E. Anthony, Adv. Mater., 2003, 15, 2009-2011.

30 S. S. Lee, M. A. Loth, J. E. Anthony and Y.-L. L. Loo, J. Am. Chem. Soc., 2012, 134, 5436-5439.

31 C. Reese and Z. Bao, Adv. Mater., 2007, 19, 4535-4538.

32 O. Ostroverkhova, D. G. Cooke, F. A. Hegmann, R. R. Tykwinski, S. R. Parkin and J. E. Anthony, Appl. Phys. Lett., 2006, 89, 192113.

33 L. Yu, X. Li, J. Smith, S. Tierney, R. Sweeney, B. K. C. C. Kjellander, G. H. Gelinck, T. D. Anthopoulos and N. Stingelin, J. Mater. Chem., 2012, 22, 9458-9461.

34 M. Jang, J. H. Park, S. Im, S. H. Kim and H. Yang, Adv. Mater., 2014, 26, 288-292.

35 K. V. Nguyen, M. M. Payne, J. E. Anthony, J. H. Lee, E. Song, B. Kang, K. Cho and W. H. Lee, Sci. Rep., 2016, 6, 33224.

36 S. S. Lee, J. M. Mativetsky, M. A. Loth, J. E. Anthony and Y.-L. Loo, ACS Nano, 2012, 6, 9879-9886.

37 A. Bolognesi, M. Berliocchi, M. Manenti, A. DiCarlo, P. Lugli, K. Lmimouni and C. Dufour, IEEE Trans. Electron Devices, 2004, 51, 1997-2003.

38 X. Li, A. Kadashchuk, I. I. Fishchuk, W. T. T. Smaal, G. Gelinck, D. J. Broer, J. Genoe, P. Heremans and H. Bässler, Phys. Rev. Lett., 2012, 108, 066601.

39 E. Yablonovitch, J. Electrochem. Soc., 1984, 131, 2625.

40 M. R. Niazi, R. Li, M. Abdelsamie, K. Zhao, D. H. Anjum, M. M. Payne, J. Anthony, D. M. Smilgies and A. Amassian, Adv. Funct. Mater., 2016, 26, 2371-2378.

41 D. J. Gundlach, J. E. Royer, S. K. Park, S. Subramanian, O. D. Jurchescu, B. H. Hamadani, A. J. Moad, R. J. Kline, L. C. Teague, O. Kirillov, C. A. Richter, J. G. Kushmerick,
L. J. Richter, S. R. Parkin, T. N. Jackson and J. E. Anthony, Nat. Mater., 2008, 7, 216-221.

42 J. E. Northrup, M. L. Tiago and S. G. Louie, Phys. Rev. B: Condens. Matter Mater. Phys., 2002, 66, 1214041-1214044.

43 F. J. Meyer zu Heringdorf, M. C. Reuter and R. M. Tromp, Nature, 2001, 412, 517-520.

44 A. Fang, A. K. Hailey, A. Grosskopf, J. E. Anthony, Y.-L. Loo and M. Haataja, APL Mater., 2015, 3, 036107.

45 K. C. Dickey, S. Subramanian, J. E. Anthony, L.-H. Han, S. Chen and Y.-L. Loo, Appl. Phys. Lett., 2007, 90, 244103.

46 K. Kim, M. Jang, M. Lee, T. K. An, J. E. Anthony, S. H. Kim, H. Yang and C. E. Park, J. Mater. Chem. C, 2016, 4, 6996-7003.

47 K. V. Nguyen, J. H. Lee, S. C. Lee, G. M. Ku and W. H. Lee, Org. Electron., 2017, 41, 107-113.

48 R. K. Hallani, K. J. Thorley, Y. Mei, S. R. Parkin, O. D. Jurchescu and J. E. Anthony, Adv. Funct. Mater., 2016, 26, 2341-2348.

49 S. Li, S. M. Ryno and C. Risko, J. Mater. Chem. C, 2018, 6, 10924-10934.

50 P. J. Diemer, J. Hayes, E. Welchman, R. Hallani, S. J. Pookpanratana, C. A. Hacker, C. A. Richter, J. E. Anthony, T. Thonhauser and O. D. Jurchescu, Adv. Electron. Mater., 2017, 3, 1600294.

51 A. K. Hailey, A. J. Petty, J. Washbourne, K. J. Thorley, S. R. Parkin, J. E. Anthony and Y.-L. Loo, Adv. Mater., 2017, 29, 1700048.

52 Y. Seo, J. H. Lee, J. E. Anthony, K. V. Nguyen, Y. H. Kim, H. W. Jang, S. Ko, Y. Cho and W. H. Lee, Adv. Mater. Interfaces, 2018, 5, 1701399.

53 D. H. Kwak, Y. Seo, J. E. Anthony, S. Kim, J. Hur, H. Chae, H. J. Park, B. Kim, E. Lee, S. Ko and W. H. Lee, Adv. Mater. Interfaces, 2020, 7, 1901696.

54 N. Nishimura, J. R. Allardice, J. Xiao, Q. Gu, V. Gray and A. Rao, Chem. Sci., 2019, 10, 4750-4760.

55 S. Arai, K. Morita, J. Tsutsumi, S. Inoue, M. Tanaka and T. Hasegawa, Adv. Funct. Mater., 2020, 30, 1906406.

56 H. Chung and Y. Diao, J. Mater. Chem. C, 2016, 4, 3915-3933.

57 M. Li, A. H. Balawi, P. J. Leenaers, L. Ning, G. H. L. Heintges, T. Marszalek, W. Pisula, M. M. Wienk, S. C. J. Meskers, Y. Yi, F. Laquai and R. A. J. Janssen, Nat. Commun., 2019, 10, 2867.

58 G. L. Schulz, F. S. U. Fischer, D. Trefz, A. Melnyk, A. HamidiSakr, M. Brinkmann, D. Andrienko and S. Ludwigs, Macromolecules, 2017, 50, 1402-1414. 